\title{
Relapse of Immune Thrombocytopenic Purpura in a Patient with COVID-19
}

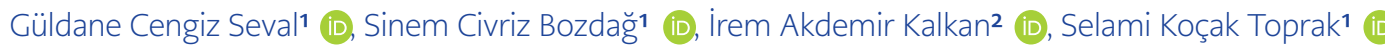 \\ Pervin Topçuoğlu ${ }^{1}$ (D), Alpay Azap² (iD, Önder Arslan ${ }^{1}$ (D) \\ 1 Department of Hematology, Ankara University School of Medicine, Ankara, Turkey \\ 2 Department of Infectious Diseases and Clinical Microbiology, Ankara University School of Medicine, Ankara, Turkey
}

To the Editor,

COVID-19, caused by severe acute respiratory syndrome coronavirus 2 (SARS-CoV-2), has been declared as a global pandemic and worldwide confirmed cases are currently approaching 76 million. And so far, thrombocytopenia has been observed in 5\% of hospitalized COVID-19 patients and $8 \%$ of those in intensive care unit $(1,2)$. Although the decrease in platelet counts was mild, confirmation of immune etiology has to be confirmed in those with severe thrombocytopenia (<20 x 109/L) or a sudden decrease in the platelet count $>50 \%$ over $24-48$ hours (3). The role of viral infections in the exacerbation of immune thrombocytopenic thrombocytopenia (ITP) has been revealed previously. We hereby report a patient who had exacerbation of ITP and was subsequently found to be COVID-19 positive.

A 34-year-old male patient has been diagnosed with ITP for fifteen years. He responded to initial steroid treatment but had to undergo splenectomy as a second-line treatment because of steroid dependency. He had lost to follow up for eight years by his sustained normalization of the platelet counts after splenectomy. He relapsed after an upper respiratory tract infection and was treated periodically with glucocorticoids and intravenous immunoglobulin (IVIG) with minimal to no response. He received azathioprine, mycophenolate mofetil, cyclophosphamide, respectively but these therapies were discontinued due to lack of response. Therefore, rituximab was administered and resulted in a rapid improvement in platelet counts. The patient regained a sustained response after rituximab for additional two years. An upper respiratory tract infection resulted in a decrease in platelet count, which resolved with eltrombopag $50 \mathrm{mg} /$ day on April 15, 2019. Since then, he had been able to maintain platelet counts consistently above $100 \times$ 109/L on eltrombopag monotherapy.

The patient was admitted to the emergency department on March 23, 2020, with a 3 -days history of fatigue, fever, dry cough, and hemoptysis. He did not report contact

\section{Corresponding Author:} Önder Arslan

E-mail: onder.arslan@medicine.ankara. edu.tr

Received: November 4, 2020 Accepted: December 28, 2020 Published: December 31, 2020

\section{Suggested citation:}

Cengiz Seval G, Civriz Bozdağ S, Akdemir Kalkan I, Koçak Toprak S, Topçuoğlu P, Azap $A$, et al. Relapse of Immune Thrombocytopenic Purpura in a Patient with Covid-19. Infect Dis Clin Microbiol 2020; 3: 184-186.

DOI: $10.36519 / \mathrm{idcm} .2020 .0026$ 
with anyone who had symptoms suspicious of COVID-19, but he had camped with a group of friends two weeks before his admission to hospital. In his physical examination, his body temperature was 390C, blood oxygen saturation was $96 \%$ at breathing room air with respiration rate $20 / \mathrm{mn}$, and he had purpuric lesions over his legs. In his complete blood count analysis platelet count was 2x109/L, hemoglobin $16 \mathrm{~g} / \mathrm{dL}$, white blood cell count was 7.4x109/L with differential as follows; $40.8 \%$ neutrophils, $36.9 \%$ lymphocytes, and $0.1 \%$ eosinophils. C-Reactive Protein and procalcitonin levels were $17.2 \mathrm{mg} / \mathrm{L}$ (range $0-5 \mathrm{mg} / \mathrm{L}$ ) and $0.084 \mathrm{ng} / \mathrm{mL}$ (range $<0.05 \mathrm{ng} / \mathrm{mL}$ ), respectively. Also, he had normal activated partial thromboplastin time, prothrombin time, and D-dimer levels. Liver function and cardiac enzyme levels were within the normal range. Throat and nasopharyngeal swab for COVID-19 reverse transcription-polymerase chain reaction (RT-PCR) was positive. Computed tomography of thorax revealed no signs of COVID-19. He has been admitted to COVID inpatient clinic for treatment. His family members were also found to be negative for SARS-Cov-2 on RT-PCR analysis.

Intravenous immune globulin was administered at a rate of $1 \mathrm{gr} / \mathrm{kg}$ BW for two days for ITP, and also eltrombopag was continued. Also, he was treated with hydroxychloroquine (200 mg twice daily, five days) and oseltamivir (75 mg twice daily, five days) as per national COVID-19 treatment guidelines at the time of diagnosis (4). His fever resolved on the first day of COVID treatment, but his cough persisted for two days and improved by the third day. His platelet count increased to $154 \times 109 / \mathrm{L}$ on the fifth day of immunoglobulin treatment. CRP decreased to normal ranges on the sixth day of treatment. Any potential side effects of hydroxychloroquine, such as vomit- ing, diarrhea, QT prolongation, and visual field disturbances were not observed during treatment.

The patient was discharged from the hospital on the sixth day of COVID treatment, when all his symptoms disappeared. He was considered for in-home isolation and home care. At the posttreatment 10th day, the SARS-CoV-2 with RT-PCR test was negative in the throat and nasopharyngeal swab. He had no symptoms and normal platelet counts on eltrombopag treatment.

The thrombocytopenia in infection-associated ITP occurs via various potential mechanisms, including increased platelet clearance due to immune complex disease, cross-reactivity of antiplatelet glycoprotein antibodies and viral antibodies. Viruses can also directly interact with platelets, altering their count and function (5).

There are very few reports on the mechanisms of thrombocytopenia in patients with COVID-19 and in most cases, the platelet counts do not decrease to a level at which bleeding occurs $(6,7)$. Zulfigar et al. reported the first case of immune thrombocytopenia with cerebral bleeding caused by SARS-CoV-2 and received intravenous immune globulin, prednisolone and eltrombopag, respectively. On day 13 of the treatment, the platelet count was 139x109/L and the patient's symptoms were disappeared (8).

In conclusion, this report aims to point out the association between COVID-19 and the exacerbation of chronic ITP. We wish to alert clinicians to this possible adverse effect of the COVID-19, underscoring the importance of careful history-taking in patients with recurrent ITP.
Informed Consent: Written consent was obtained from the patient.

Peer-review: Externally peer-reviewed

Conflict of Interest: The author have no conflict of interest to declare.
Financial Disclosure: The authors declared that this study has received no financial support. 


\section{REFERENCES}

1 Guan WJ, Ni ZY, Hu Y, Liang WH, Ou CQ, He JX, et al. Clinical Characteristics of Coronavirus Disease 2019 in China. N Engl J Med 2020; 382: 1708-20.

2 Huang C, Wang Y, Li X, Ren L, Zhao J, Hu Y, et al. Clinical features of patients infected with 2019 novel coronavirus in Wuhan, China. Lancet 2020; 395: 497-506.

3 Pavord S, Thachil J, Hunt BJ, Murphy M, Lowe G, Laffan M, et al. Practical guidance for the management of adults with immune thrombocytopenia during the COVID-19 pandemic. Br J Haematol 2020; 189: 1038-43.

4 Republic of Turkey Ministry of Health. Management and medical treatment of adults with COVID-19, March 2020. Available from: URL: https://hsgm.saglik.gov.tr/tr/
5 Stasi R, Willis F, Shannon MS, Gordon-Smith EC. Infectious causes of chronic immune thrombocytopenia. Hematol Oncol Clin North Am 2009; 23:1275-97.

6 Xu P, Zhou Q, Xu J. Mechanism of thrombocytopenia in COVID-19 patients. Ann Hematol. 2020; 99 (6): 1205-8.

7 Lippi G, Plebani M, Henry BM. Thrombocytopenia is associated with severe coronavirus disease 2019 (COVID-19) infections: a meta-analysis. Clin Chim Acta. 2020; 506: 145-148.

8 Zulfiqar AA, Villalba NL, Hassler P, Andres E. Immune thrombocytopenic purpura in a patient with COVID-19. N Engl J Med. 2020; 382: e43. 